\title{
Countable contraction mappings in metric spaces: invariant sets and measure
}

\author{
María Fernanda Barrozo ${ }^{1,2 *}$, Ursula Molter $r^{3,4 \dagger}$ \\ 1 Departamento de Matemática, Facultad de Ciencias Físico-Matemáticas y Naturales, Universidad Nacional de San Luis, \\ Ejército de Los Andes 950, 5700 San Luis, Argentina \\ 2 IMASL-CONICET, Italia 1556, 5700 San Luis, Argentina \\ 3 Departamento de Matemática, Facultad de Ciencias Exactas y Naturales, Universidad de Buenos Aires, Ciudad Universitaria, \\ Pabellón I, 1428 Capital Federal, Argentina \\ 4 IMAS-CONICET, Ciudad Universitaria, Pabellón 1, 1428 Buenos Aires, Argentina
}

Received 21 January 2013 ; accepted 6 August 2013

Abstract: We consider a complete metric space $(X, d)$ and a countable number of contraction mappings on $X, \mathcal{F}=\left\{F_{i}\right.$ : $i \in \mathbb{N}\}$. We show the existence of a smallest invariant set (with respect to inclusion) for $\mathcal{F}$. If the maps $F_{i}$ are of the form $F_{i}(\mathbf{x})=r_{i} \mathbf{x}+\mathbf{b}_{i}$ on $X=\mathbb{R}^{d}$, we prove a converse of the classic result on contraction mappings, more precisely, there exists a unique bounded invariant set if and only if $r=\sup _{i} r_{i}$ is strictly smaller than 1 .

Further, if $\rho=\left\{\rho_{k}\right\}_{k \in \mathbb{N}}$ is a probability sequence, we show that if there exists an invariant measure for the system $(\mathcal{F}, \rho)$, then its support must be precisely this smallest invariant set. If in addition there exists any bounded invariant set, this invariant measure is unique, even though there may be more than one invariant set.

MSC: $\quad 28 \mathrm{~A} 80,37 \mathrm{C} 25,37 \mathrm{C} 70$

Keywords: Contraction maps $\cdot$ Countable iterated function system $\bullet$ Invariant set $\bullet$ Invariant measure (C) Versita Sp. z o.o.

\section{Introduction}

A map $F$ from a metric space $(X, d)$ into itself is a contraction, if there exists a constant $c, 0<c<1$, such that $d(F(x), F(y)) \leq c d(x, y)$, for all $x, y \in X$. We denote by Lip $(F)$ the smallest of all such constants and we call it the Lipschitz constant or contraction factor of $F$.

* E-mail: mfbarroz@unsl.edu.ar

† E-mail:umolter@dm.uba.ar 
In [6], Hutchinson introduced the notion of invariant set and invariant measure for a finite set of contraction mappings from a complete metric space $(X, d)$ into itself. In particular, he proved the following now classical results.

Theorem 1.1 ([6]).

Let $\mathcal{F}=\left\{F_{1}, \ldots, F_{N}\right\}$ be a finite family of contraction mappings in the complete metric space $(X, d)$. Let $\mathcal{C}(X)$ be the set of non-empty closed and bounded subsets of $X$, and let $\rho_{1}, \ldots, \rho_{N} \in(0,1)$ and $\sum_{i=1}^{N} \rho_{i}=1$. Then we have:

- There exists a unique set $K \in \mathcal{C}(X)$ that is invariant with respect to $\mathcal{F}$, i.e.,

$$
K=\overline{\bigcup_{i=1}^{N} F_{i}(K)} .
$$

$K$ is in fact compact and is the closure of the set of fixed points of all finite compositions of elements of $\mathcal{F}$.

- There exists a unique Borel regular (outer) measure $\mu$ with bounded support, and total mass 1 , that is invariant with respect to $(\mathcal{F}, \rho)$, i.e.,

$$
\mu=\sum_{i=1}^{N} \rho_{i} F_{i \#} \mu,
$$

where $F_{i \#} \mu$ is the measure defined by $F_{i \#} \mu(E)=\mu\left(F_{i}^{-1}(E)\right)$ for each $E \subset X$.

- The support of $\mu, \operatorname{supp} \mu$, is precisely the invariant set $K$.

Similar results can be found in [2]. For general references see [3, 4, 8].

If instead of a finite number of contraction mappings one considers a countable collection, the notions of invariant set and invariant measure can be extended in a natural way.

\section{Definition 1.2.}

Let $\mathcal{F}=\left\{F_{i}\right\}_{i=1}^{\infty}$ be a countable family of contraction mappings in the complete metric space $(X, d)$. We say that a non-empty set $E \subset X$ is an invariant set for $\mathcal{F}$ if

$$
E=\overline{\bigcup_{i=1}^{\infty} F_{i}(E) .}
$$

If $\rho=\left(\rho_{1}, \rho_{2}, \ldots\right)$ is a probability sequence, i.e. $\rho_{i} \in(0,1)$ and $\sum_{i=1}^{\infty} \rho_{i}=1$, we say that an outer measure $\mu$ is an invariant measure for $(\mathcal{F}, \rho)$ if

$$
\mu=\sum_{i=1}^{\infty} \rho_{i} F_{i \#} \mu,
$$

where $F_{i \#} \mu$ is (as before) the measure defined by $F_{i \# \mu}(E)=\mu\left(F_{i}^{-1}(E)\right)$ for each $E \subset X$.

Finite families of contraction mappings automatically satisfy two conditions which allow to ensure the existence and uniqueness of a bounded invariant set: on one hand, the boundedness of the set of fixed points, and, on the other hand, the fact that the maximum of Lipschitz constants is strictly less than 1. In general, if one has a countable system these conditions are not automatically satisfied.

\section{Definition 1.3.}

A set $\mathcal{F}=\left\{F_{i}\right\}_{i \in l}$, for $I \subset \mathbb{N}$ either finite or infinite, in a complete metric space $(X, d)$, where $F_{i}$ are contraction mappings, is called Iterated Function System (IFS). We denote by $r$ the supremum of the contraction factors, and by $D$ the set of fixed points, i.e.,

$$
r=\sup _{i \in I}\left\{r_{i}: r_{i} \text { is the contraction factor of } F_{i}\right\}, \quad D=\left\{x_{i}: F_{i}\left(x_{i}\right)=x_{i}, i \in I\right\} .
$$


In the case that $D$ is bounded and $r<1$, Bandt [1] showed the existence and uniqueness of a bounded invariant set for $\mathcal{F}$, where $\mathcal{F}=\left\{F_{i}\right\}_{i=1}^{\infty}$ is a countable family of contraction mappings. Countable iterated function systems were first introduced by Mauldin and Williams ([11], see also $[9,10])$.

In the present article we show that for any countable family of contraction mappings $\mathcal{F}$ there exists an invariant set (see also [13]). In fact, there exists a smallest invariant set, with respect to inclusion, for $\mathcal{F}$. We show that this set is the closure of the set of fixed points of finite compositions of members of $\mathcal{F}$. For this result we do not need to assume that $D$ is bounded neither that $r<1$. It follows that the bounded invariant set obtained by Bandt is, as in the finite case, the closure of the set of fixed points of finite compositions of members of $\mathcal{F}$.

We further show that boundedness of $D$ is necessary for the existence of a bounded invariant set. In fact, since any invariant set contains $D$, if there exists a bounded invariant set then $D$ is bounded. However, we show that the condition $r<1$ is not necessary for existence of a bounded invariant set.

Further, the condition that $D$ be bounded is not sufficient: we exhibit an example in which the set of fixed points is bounded, but there does not exist a bounded invariant set. However, if $X=\mathbb{R}$ and the system contains only nondecreasing functions, boundedness of $D$ does suffice (cf. Theorem 2.7).

In addition, we prove a kind of converse to the theorem by Bandt in [1]: under certain restrictions, if there exists a unique bounded invariant set, then necessarily $r<1$ (cf. Theorem 2.10).

Finally, we prove that the support of any invariant measure for the countable IFS $(\mathcal{F}, \rho)$, where $\rho$ is a probability sequence, must coincide precisely with the smallest invariant set that we showed to exist. We further show, that if there exists a bounded invariant set, then the invariant measure exists and is unique, even though the invariant set might not be unique.

\section{Invariant sets}

Let $\mathcal{F}=\left\{F_{i}: i \in \mathbb{N}\right\}$ be a countable family of contraction mappings in the complete metric space $(X, d)$. First, we will prove that there exists a smallest invariant set for $\mathcal{F}$, with respect to inclusion: the closure of the set of fixed points of finite compositions of members of $\mathcal{F}$.

As before, if $\mathcal{F}=\left\{F_{i}: i \in \mathbb{N}\right\}$ is a countable family of contraction mappings in the complete metric space $(X, d)$, $x_{i}$ will denote the fixed point of $F_{i}$ and $D$ will be the set of fixed points of members of $\mathcal{F}$. For all $i \in \mathbb{N}, r_{i}$ will denote the Lipschitz constant of $F_{i}$, i.e., $r_{i}=\operatorname{Lip}\left(F_{i}\right)$, and $r=\sup _{i} r_{i}$. Further, $F_{i_{1} \ldots i_{k}}$ will denote the composition $F_{i_{1}} \circ \cdots \circ F_{i_{k}}, x_{i_{1} \ldots i_{k}}$ will be the fixed point of $F_{i_{1} \ldots i_{k}}$ and $P$ will be the set of fixed points of finite compositions of members of $\mathcal{F}$.

We first need the following result, which is analogous to the finite IFS case.

\section{Lemma 2.1.}

Let $\mathcal{F}=\left\{F_{i}: i \in \mathbb{N}\right\}$ be a countable family of contraction mappings in the complete metric space $(X, d)$ and let $P$ be the set of fixed points of finite compositions of members of $\mathcal{F}$. If $A \subset X$ is a non-empty closed set such that $F_{i}(A) \subset A$ for all $i \in \mathbb{N}$, then $P \subset A$.

Proof. Let $x_{i_{1} \ldots i_{p}}$ be the fixed point of $F_{i_{1} \ldots i_{p}}$, and let $a \in A$. Then

$$
\lim _{k \rightarrow \infty} F_{i_{1} \ldots i_{p}}^{k}(a)=x_{i_{1} \ldots i_{p}}
$$

Since $F_{i}(A) \subset A$ for all $i \in \mathbb{N}, F_{i_{1} \ldots i_{p}}(a) \in A$, then we have $F_{i_{1} \ldots i_{p}}^{k}(a) \in A$ for all $k$. Since $A$ is closed, $x_{i_{1} \ldots i_{p}} \in A$. Hence, $P \subset A$.

\section{Theorem 2.2.}

Let $\mathcal{F}=\left\{F_{i}: i \in \mathbb{N}\right\}$ be a countable family of contraction mappings in the complete metric space $(X, d)$. If $P$ is the set of fixed points of finite compositions of members of $\mathcal{F}$, then $\bar{P}$ is the smallest invariant set for $\mathcal{F}$, with respect to inclusion. 
Proof. First, we will prove that $\bar{P}$ is an invariant set for $\mathcal{F}$. Note that for a fixed $N \in \mathbb{N}$, if we consider the finite subfamily $\left\{F_{1}, \ldots, F_{N}\right\}$, and let $P_{N}$ be the set of fixed points of finite compositions of $F_{i}$ with $1 \leq i \leq N$, from Theorem 1.1 it follows that $\overline{P_{N}}$ is the unique compact invariant set for the IFS $\left\{F_{1}, \ldots, F_{N}\right\}$.

In order to prove the inclusion $\overline{U F_{i}(\bar{P})} \subset \bar{P}$, it is enough to show that $F_{i}(P) \subset \bar{P}$ for all $i$. For this, let $i \in \mathbb{N}$ be fixed, and let $x_{\alpha_{1} \ldots \alpha_{n}} \in P$ be the fixed point of $F_{\alpha_{1} \ldots \alpha_{n}}$. We need to show that $F_{i}\left(x_{\alpha_{1} \ldots \alpha_{n}}\right) \in \bar{P}$. We define the following sequence $\left\{y_{k}\right\}_{k \in \mathbb{N}}$ in $P$ : for each $k$, let $y_{k}$ be the fixed point of $F_{i} \circ F_{\alpha_{1} \ldots \alpha_{n}}^{k}$. So $y_{1}=x_{i \alpha_{1} \ldots \alpha_{n}}, y_{2}=x_{i \alpha_{1} \ldots \alpha_{n} \alpha_{1} \ldots \alpha_{n}}$, etc. If $N=\max \left\{\alpha_{1}, \ldots, \alpha_{n}, i\right\}$, then $x_{\alpha_{1} \ldots \alpha_{n}} \in P_{N}$ and $y_{k} \in P_{N}$ for all $k$. Therefore,

$$
d\left(y_{k}, F_{i}\left(x_{\alpha_{1} \ldots \alpha_{n}}\right)\right) \leq r_{i} d\left(F_{\alpha_{1} \ldots \alpha_{n}}^{k}\left(y_{k}\right), x_{\alpha_{1} \ldots \alpha_{n}}\right)<\left(r_{\alpha_{1}} \cdots r_{\alpha_{n}}\right)^{k} \operatorname{diam} P_{N}
$$

Hence, since $r_{\alpha_{1}} \cdots r_{\alpha_{n}}<1$ and diam $P_{N}<\infty$, we have

$$
\lim _{k \rightarrow \infty} y_{k}=F_{i}\left(x_{\alpha_{1} \ldots \alpha_{n}}\right)
$$

which implies that $F_{i}\left(x_{\alpha_{1} \ldots \alpha_{n}}\right) \in \bar{P}$, as we wanted to show.

For the other inclusion we will show that $P \subset \cup F_{i}(\bar{P})$. Let $x_{\alpha_{1} \ldots \alpha_{n}} \in P$ and consider the sequence $\left\{z_{k}\right\}_{k \in \mathbb{N}}$, where $z_{k}$ is the fixed point of the composition $F_{\alpha_{2} \ldots \alpha_{n}} \circ F_{\alpha_{1} \ldots \alpha_{n}}^{k}$. As before, if $N=\max \left\{\alpha_{1}, \ldots, \alpha_{n}\right\}$ then $x_{\alpha_{1} \ldots \alpha_{n}} \in P_{N}$ and $z_{k} \in P_{N}$ for all $k$. Consequently,

$$
d\left(z_{k}, F_{\alpha_{2} \ldots \alpha_{n}}\left(x_{\alpha_{1} \ldots \alpha_{n}}\right)\right) \leq r_{\alpha_{2}} \cdots r_{\alpha_{n}}\left(r_{\alpha_{1}} \cdots r_{\alpha_{n}}\right)^{k} d\left(z_{k}, x_{\alpha_{1} \ldots \alpha_{n}}\right)<\left(r_{\alpha_{1}} \cdots r_{\alpha_{n}}\right)^{k} \operatorname{diam} P_{N},
$$

which implies $\lim _{k \rightarrow \infty} z_{k}=F_{\alpha_{2} \ldots \alpha_{n}}\left(x_{\alpha_{1} \ldots \alpha_{n}}\right) \in \bar{P}$ and therefore

$$
x_{\alpha_{1} \ldots \alpha_{n}}=F_{\alpha_{1}}\left(F_{\alpha_{2} \ldots \alpha_{n}}\left(x_{\alpha_{1} \ldots \alpha_{n}}\right)\right) \in F_{\alpha_{1}}(\bar{P})
$$

Thus, the closure of $P$ is an invariant set for $\mathcal{F}$.

In order to show that $\bar{P}$ is the smallest invariant set, let $A$ be an invariant set for $\mathcal{F}$. By Definition 1.2, $A$ is non-empty and closed and satisfies $F_{i}(A) \subset A$ for all $i \in \mathbb{N}$. By Lemma 2.1 we obtain that $P \subset A$, therefore $\bar{P} \subset A$.

\section{Remark 2.3.}

Notice that in the previous theorem we do not assume that $D$ is bounded neither that $r<1$. Therefore, for any countable family of contraction mappings there exists an invariant set.

\section{Remark 2.4.}

The assertion that $\bar{P}$ is an invariant set can also be obtained using Hutchinson's Theorem 1.1 and a result in [13].

Recalling the results of Bandt [1], which show that if $r<1$ and $D$ is bounded, there exists a unique closed and bounded invariant set with respect to $\mathcal{F}$; using Theorem 2.2, we conclude that this unique set must necessarily coincide with $\bar{P}$ (see also [5, 12]). Note that Bandt also showed that this set is not necessarily compact. This extends completely the result of Hutchinson to the countable IFS case.

\section{Corollary 2.5.}

Let $\mathcal{F}=\left\{F_{i}: i \in \mathbb{N}\right\}$ be a countable family of contraction mappings in the complete metric space $(X, d)$. If $r=$ $\sup _{i \in \mathbb{N}} \operatorname{Lip}\left(F_{i}\right)<1$ and $D$, the set of fixed points of elements of $\mathcal{F}$, is bounded, then $\bar{P}$ is the unique bounded invariant set for $\mathcal{F}$, where $P$ is the set of fixed points of finite compositions of members of $\mathcal{F}$.

From Theorem 2.2 it follows that boundedness of $D$ is a necessary condition for the existence of a bounded invariant set. Indeed, since every invariant set contains $D$, if there exists a bounded invariant set, then $D$ must be bounded. On the other hand, the next example shows that the condition $r<1$ is not necessary. 


\section{Example 2.6.}

For every $i \in \mathbb{N}$, define $F_{i}: \mathbb{R} \rightarrow \mathbb{R}$,

$$
F_{i}(x)=\frac{i}{i+1} x+\frac{1}{(i+1)^{2}}
$$

Then, the set of fixed points $D=\{1 /(i+1)\}_{i \in \mathbb{N}}$ is bounded, but $\sup _{i} r_{i}=1$. However, there exists a bounded invariant set, for example, $[0,1 / 2]$. Indeed, for every $a \leq 0$ and $b \geq 1 / 2$, the closed interval $[a, b]$ is an invariant set.

The previous example can be extended for every countable family of non-decreasing contraction mappings in $\mathbb{R}$.

\section{Theorem 2.7.}

Let $\mathcal{F}=\left\{F_{i}: i \in \mathbb{N}\right\}$ be a countable family of contraction mappings in $\mathbb{R}$, such that every $F_{i}$ is non-decreasing. If the set of fixed points of members of $\mathcal{F}$ is bounded, then there exists a bounded invariant set for $\mathcal{F}$.

Proof. Let $D$ be the set of fixed points of members of $\mathcal{F}$, and let us consider $\alpha=\inf D$ and $\beta=\sup D$. We will show that the interval $I=[\alpha, \beta]$ satisfies $F_{i}(I) \subset I$ for all $i \in \mathbb{N}$. Since every $F_{i}$ is continuous and non-decreasing, we have that $F_{i}(I)=\left[F_{i}(\alpha), F_{i}(\beta)\right]$. Further, since $F_{i}$ is contractive, $F_{i}(\beta)-F_{i}\left(x_{i}\right)<\beta-x_{i}$ and $F_{i}\left(x_{i}\right)-F_{i}(\alpha)<x_{i}-\alpha$. Therefore $\alpha<F_{i}(\alpha) \leq F_{i}(\beta)<\beta$. Thus, $F_{i}(I) \subset l$. The conclusion now follows from Lemma 2.1.

However, in a general case, boundedness of $D$ is not a sufficient condition for the existence of a bounded invariant set, since it can be the case (as we show below) that $D$ is bounded, but $P$ is not.

\section{Example 2.8.}

Consider the contraction mappings in $\mathbb{R}$ defined by

$$
F_{i}(x)=-\frac{i}{i+1} x+\frac{2 i+1}{i}, \quad \widetilde{F}_{i}(x)=-\frac{i}{i+1} x+\frac{1}{i+1}, \quad i \in \mathbb{N}
$$

Let $\mathcal{F}$ be the countable family $\mathcal{F}=\left\{F_{i}: i \in \mathbb{N}\right\} \cup\left\{\widetilde{F}_{i}: i \in \mathbb{N}\right\}$. The set of fixed points is contained in $[0,2]$. However, there does not exist any bounded invariant set for $\mathcal{F}$.

To see this, we consider the compositions $\widetilde{F}_{i} \circ F_{i}$ and $F_{i} \circ \widetilde{F}_{i}, i \in \mathbb{N}$, and look at the set $\left\{y_{i}: \widetilde{F}_{i} \circ F_{i}\left(y_{i}\right)=y_{i}\right\} \cup\left\{z_{i}:\right.$ $\left.F_{i} \circ \tilde{F}_{i}\left(z_{i}\right)=z_{i}\right\}$. A simple computation shows that the fixed point of $\tilde{F}_{i} \circ F_{i}$ is $y_{i}=-2 i(i+1) /(2 i+1)$ and the fixed point of $F_{i} \circ \widetilde{F}_{i}$ is $z_{i}=\left(-i^{2}+(i+1)^{2}(2 i+1)\right) /(i(2 i+1))$. Thus, the set $P$ cannot be bounded.

We will conclude this section proving a kind of converse to the theorem by Bandt. In some cases we will be able to prove that, if there exists a unique bounded invariant set, then necessarily $r<1$. We begin by proving a general lemma about enlargements of an invariant set $A$. Recall that the $\varepsilon$-enlargement of a set $A$ in a metric space $(X, d)$ is defined by

$$
A_{\varepsilon}=\{y \in X: d(y, A)<\varepsilon\} .
$$

\section{Lemma 2.9.}

Let $\mathcal{F}=\left\{F_{i}: i \in \mathbb{N}\right\}$ be a countable family of contraction mappings in a complete metric space $(X, d)$, and let $A$ an invariant set for $\mathcal{F}$. If $\alpha>0$, then $F_{i}\left(A_{\alpha}\right) \subset A_{\alpha}$ for all $i$.

Proof. Let $x \in A_{\alpha}$. Then, by definition of $A_{\alpha}$, there is $y \in A$ such that $d(x, y)<\alpha$. Since $A$ is invariant, $F_{i}(y) \in A$. Moreover, $d\left(F_{i}(x), F_{i}(y)\right) \leq r_{i} \cdot d(x, y)<\alpha$ and therefore $F_{i}(x) \in A_{\alpha}$.

For $X=\mathbb{R}$, and $F_{i}$ similarities, we can sharpen the previous result. 
Theorem 2.10.

Let $\mathcal{F}=\left\{F_{i}: i \in \mathbb{N}\right\}$ be a countable family of contractive similarities in $\mathbb{R}\left(\left|F_{i}(x)-F_{i}(y)\right|=r_{i}|x-y|\right.$ for all $x, y \in \mathbb{R}$, $\left.r_{i}<1\right)$, such that $r=\sup _{i} r_{i}=1$. If there exists a bounded set $A$ that is invariant for $\mathcal{F}$, then there exists $\alpha>0$ such that $\overline{A_{\alpha}}$ is invariant. (Hence if a bounded invariant set exists, it is not unique, in contrast to the case $r<1$ !)

Proof. Let $\alpha>0$ be such that $A_{\alpha}=(a, b)=I$ for some interval $I$. By the previous lemma, we have that $F_{i}(I) \subset I$ for each $i$. Then $\overline{\bigcup_{i=1}^{\infty} F_{i}(\bar{l})} \subset \bar{l}$.

For the other inclusion, let $x \in I$ and let $\delta=\max \{b-x, x-a\}$. Now choose $r_{i}>\delta /(b-a)$, which is possible, since $r=1$. Since each $F_{i}$ is a similarity, $F_{i}(l)$ is an interval either $\left(F_{i}(a), F_{i}(b)\right)$ or $\left(F_{i}(b), F_{i}(a)\right)$ depending on the monotonicity of $F_{i}$. If $F_{i}$ is increasing, $F_{i}(b)-F_{i}(a)=r_{i}(b-a)>\delta$. This implies that $F_{i}(b)-F_{i}(a)>b-x$ and $F_{i}(b)-F_{i}(a)>x-a$, and consequently

$$
x-F_{i}(a)>b-F_{i}(b)>0 \quad \text { and } \quad F_{i}(b)-x>F_{i}(a)-a>0 .
$$

Hence $F_{i}(a)<x<F_{i}(b)$ and so $x \in F_{i}(I)$. If in turn $F_{i}$ is decreasing, an analogous reasoning allows us to conclude that, $x \in F_{i}(I)$. Hence $\bar{l} \subset \overline{\bigcup_{i=1}^{\infty} F_{i}(\bar{l})}$.

As a corollary we have a converse to the theorem of Bandt.

Corollary 2.11.

Let $\mathcal{F}=\left\{F_{i}: i \in \mathbb{N}\right\}$ be a countable family of contractive similarities in $\mathbb{R}$. If there exists a unique bounded invariant set for $\mathcal{F}$, then $r=\sup _{i} \operatorname{Lip}\left(F_{i}\right)<1$.

The previous theorem can be extended to contractive similarities in $\mathbb{R}^{n}$ that are multiples of the identity, i.e. $F_{i}: \mathbb{R}^{n} \rightarrow \mathbb{R}^{n}$, $F_{i}(\mathbf{x})=r_{i} \mathbf{x}+\mathbf{b}_{i},\left|r_{i}\right|<1$.

Theorem 2.12.

Let $\mathcal{F}=\left\{F_{i}: i \in \mathbb{N}\right\}$, with $F_{i}: \mathbb{R}^{n} \rightarrow \mathbb{R}^{n}, F_{i}(\mathbf{x})=r_{i} \mathbf{x}+\mathbf{b}_{i},\left|r_{i}\right|<1$. Let $r=\sup \left|r_{i}\right|$, and $P$ be the subset of $\mathbb{R}^{n}$ of fixed points of finite compositions of $\left\{F_{i}\right\}_{i \in \mathbb{N}}$. If $P$ is bounded and $r=1$, then there exists a rectangle $R=I_{1} \times \cdots \times I_{n}$ such that $P \subsetneq R$ that is invariant for $\mathcal{F}$.

Proof. We write $\mathbf{x}=\left(x_{1}, \ldots, x_{n}\right)$ and $\mathbf{b}_{i}=\left(b_{i 1}, \ldots, b_{i n}\right)$, thus

$$
F_{i}(\mathbf{x})=r_{i} \mathbf{x}+\mathbf{b}_{i}=\left(r_{i} x_{1}+b_{i 1}, \ldots, r_{i} x_{n}+b_{i n}\right)
$$

and we call $f_{i j}$ the maps from $\mathbb{R}$ to $\mathbb{R}$ defined by the $j$-th coordinate $\left(f_{i j}\left(x_{j}\right)=r_{i} x_{j}+b_{i j}\right)$, so $F_{i}(x)=\left(f_{i 1}\left(x_{1}\right), \ldots, f_{i n}\left(x_{n}\right)\right)$ and $f_{j}$ is a contractive similarity in $\mathbb{R}$, of contraction factor $r_{i}$, for $j=1, \ldots, n$.

We have that $\mathrm{x}$ is a fixed point for $F_{i}$ if and only if $x_{j}$ is a fixed point for $f_{i j}$ for $j=1, \ldots, n$. Now, for every $j=1, \ldots, n$, let $\mathcal{F}_{j}=\left\{f_{i j}\right\}_{i \in \mathbb{N}}$ be the countable IFS on the line, defined by the "coordinate" maps of $\mathcal{F}$. If $P_{j}$ is the set of fixed points of finite compositions of the maps from $\mathcal{F}_{j}$, by our assumption we have that $\bar{P}_{j}$ is bounded and invariant for $\mathcal{F}_{j}$ for $j=1, \ldots, n$. By the proof of Theorem 2.10, there exist intervals $I_{j}$ such that

- $\bar{P}_{j} \subsetneq I_{j}$, and therefore $P \subsetneq R=I_{1} \times \cdots \times I_{n}$,

- $f_{i j}\left(l_{j}\right) \subset l_{j}$ for all $i$.

Hence, since $F_{i}=r_{i} \mathrm{ld}+\mathbf{b}_{i}$ we have that $F_{i}(R)=f_{i 1}\left(I_{1}\right) \times \cdots \times f_{i n}\left(I_{n}\right) \subset I_{1} \times \cdots \times I_{n}=R$ for all $i$.

Let now $\mathrm{x} \in R$ (i.e. $x_{j} \in I_{j}, 1 \leq j \leq n$ ). If $I_{j}=\left[\alpha_{j}, \beta_{j}\right]$ we take $\delta_{j}=\max \left\{\beta_{j}-x_{j}, x_{j}-\alpha_{j}\right\}$. Since $r=1$, we choose $i \in \mathbb{N}$ such that $r_{i}>\max \left\{\delta_{j} /\left(\beta_{j}-\alpha_{j}\right): 1 \leq j \leq n\right\}$. From the proof of Theorem 2.10 it follows that for each $j, 1 \leq j \leq n$, $x_{j} \in f_{i j}\left(I_{j}\right)$. Hence $\mathrm{x} \in f_{i 1}\left(I_{1}\right) \times \cdots \times f_{i n}\left(I_{n}\right)=F_{i}(R)$. So $R$ is invariant for $\mathcal{F}$. 
We again have the same corollary.

\section{Corollary 2.13.}

Let $\mathcal{F}=\left\{F_{i}: i \in \mathbb{N}\right\}$ be a countable family of contractive similarities in $\mathbb{R}^{n}$, such that $F_{i}(\mathbf{x})=r_{i} \mathbf{x}+\mathbf{b}_{i}$, $\left|r_{i}\right|<1$. If there exists a unique bounded invariant set for $\mathcal{F}$, then $r=\sup \left|r_{i}\right|<1$.

\section{Invariant measures}

In addition to providing a complete proof of Hutchinson's theorem for the most general case, Kravchenko in [7] generalized Hutchinson's Theorem 1.1 to the case of a countable set of maps and gave a sufficient condition for the existence and uniqueness of an invariant measure. We first need to recall the following definition.

\section{Definition 3.1.}

A measure $v$ is separable if there exists a separable Borel set $A \subset X$ such that $v(X \backslash A)=0$.

Note that if $v$ is a finite measure, $v$ is separable if and only if $v(X \backslash \operatorname{supp} v)=0$.

Theorem 3.2 ([7]).

Let $(X, d)$ be a complete metric space. Let $\mathcal{F}=\left\{F_{i}: i \in \mathbb{N}\right\}$ be a countable family of contraction mappings with fixed points $x_{i}$. Let $\rho=\left\{\rho_{i}\right\}_{i \in \mathbb{N}}$, be a probability sequence, i.e. $0<\rho_{i}<1$ and $\sum_{i=1}^{\infty} \rho_{i}=1$. If $\sum_{i=1}^{\infty} \rho_{i} d\left(x_{1}, x_{i}\right)<\infty$, then there exists a unique measure $\mu \in M_{\mathrm{s}}(X)$ that is invariant with respect to $(\mathcal{F}, \rho)$, i.e.

$$
\mu=\sum_{i \in \mathbb{N}} \rho_{i} F_{i \#} \mu .
$$

Here $M_{\mathrm{s}}(X)$ is the space of all separable probability measures that satisfy $\int f d \mu<\infty$ for all $f: X \rightarrow \mathbb{R}$ with finite Lipschitz constant.

Note that the hypotheses of this theorem are slightly weaker than the ones of Bandt, since $r$ is not required to be strictly smaller than 1 . Moreover, if $D$ is bounded and $\sum_{i=1}^{\infty} \rho_{i}=1$ we have

$$
\sum_{i} \rho_{i} d\left(x_{1}, x_{i}\right) \leq \sum_{i} \rho_{i} \operatorname{diam} D=\operatorname{diam} D<\infty
$$

Hence, in the case of bounded $D$, for each (countable) probability sequence we have a unique measure in $M_{\mathrm{s}}(X)$ that is invariant with respect to $(\mathcal{F}, \rho)$ (independently of the value of $r$ ).

\section{Remark 3.3.}

In [12] Mihail and Miculescu worked with the Infinite Iterated Function System under the same hypothesis as Bandt in [1] from a different viewpoint. They showed that for IIFS, the unique invariant set for $\mathcal{F}$ (which is bounded because of the hypothesis on IIFS) coincides with the closure of the canonical projection of the shift space.

In this case, analogously to the finite case, one has immediately that the unique invariant measure is $\pi_{\#} \tau$, and that the support of this measure is the unique invariant set for $\mathcal{F}$ (here $\tau$ is the product measure on $\mathbb{N}^{\mathbb{N}}$ induced by $\rho(i)=\rho_{i}$ on each factor).

Their results rely strongly on the fact that the set of fixed points is bounded and the supremum of the Lipschitz constants of the system is strictly smaller than 1. 
We will prove next that the support of any invariant measure for $(\mathcal{F}, \rho)$ must coincide with the smallest invariant set for $\mathcal{F}$, where $\rho$ is a probability sequence, even for the case $r=1$. We start proving that if $\mu$ is an invariant measure for $(\mathcal{F}, \rho)$ then its support is an invariant set for $\mathcal{F}$. We first need the following result.

\section{Lemma 3.4.}

Let $(X, d)$ be a metric space. If $F: X \rightarrow X$ is a Lipschitz map and $\mu$ is a measure in $X$, then $F(\operatorname{supp} \mu) \subset \operatorname{supp} F_{\# \mu}$.

Proof. Let us consider $y=F(x)$ for some $x \in \operatorname{supp} \mu$. In order to prove that $y \in \operatorname{supp} F_{\#} \mu$, we need to show that any ball centered at $y$ has positive $F_{\#} \mu$-measure. We take $\varepsilon>0$ and consider the ball $B(x, \delta)=\{z \in X: d(x, z)<\delta\}$, with $\delta=r^{-1} \varepsilon$ where $r=\operatorname{Lip}(F)$. Since $F$ is Lipschitz,

$$
F(B(x, \delta)) \subset B(F(x), r \delta)=B(y, \varepsilon),
$$

and then, $B(x, \delta) \subset F^{-1}(B(y, \varepsilon))$. Hence,

$$
F_{\#} \mu(B(y, \varepsilon))=\mu\left(F^{-1}(B(y, \varepsilon))\right) \geq \mu(B(x, \delta))>0
$$

because $x \in \operatorname{supp} \mu$. Thus $y \in \operatorname{supp} F_{\# \mu}$.

We are now ready to prove the announced theorem.

\section{Theorem 3.5.}

Let $(X, d)$ be complete metric space. Let $\mathcal{F}=\left\{F_{i}: i \in \mathbb{N}\right\}$ be a countable family of contraction mappings on $X$ and let $\rho=\left\{\rho_{i}: i \in \mathbb{N}\right\}$ be a probability sequence, i.e., $0<\rho_{i}<1$ and $\sum_{i=1}^{\infty} \rho_{i}=1$. If $\mu$ is an invariant measure for $(\mathcal{F}, \rho)$, then the support of $\mu$ is an invariant set for $\mathcal{F}$.

Proof. Assume that $\mu$ is an invariant measure for $(\mathcal{F}, \rho)$ and let $A=\operatorname{supp} \mu$. Then, by Definition 1.2

$$
\mu=\sum_{i=1}^{\infty} \rho_{i} F_{i \#} \mu
$$

where $F_{i \#} \mu$ is the measure defined by $F_{i \#} \mu(E)=\mu\left(F_{i}^{-1}(E)\right)$ for each $E \subset X$. By Lemma 3.4 we have that $F_{i}(A) \subset$ supp $F_{i \#} \mu$ for all $i$. Further, it is clear that

$$
\operatorname{supp} F_{i \#} \mu \subset \operatorname{supp}\left(\sum_{i=1}^{\infty} \rho_{i} F_{i \#} \mu\right)=\operatorname{supp} \mu=A \text {. }
$$

Hence, $\bigcup_{i=1}^{\infty} F_{i}(A) \subset A$. Since $A$ is closed, we obtain $\overline{\bigcup_{i=1}^{\infty} F_{i}(A)} \subset A$. On the other hand, let $a \in A$ and $\varepsilon>0$. Since $\mu(B(a, \varepsilon))>0$ and $\mu=\sum_{i=1}^{\infty} \rho_{i} F_{i \#} \mu$, there must exist $i$ such that $\mu\left(F_{i}^{-1}(B(a, \varepsilon))\right)>0$. Consequently,

$$
F_{i}^{-1}(B(a, \varepsilon)) \cap A \neq \emptyset .
$$

That is, there exists $x \in A$ such that $d\left(F_{i}(x), a\right)<\varepsilon$. Then $a \in \overline{\bigcup_{i=1}^{\infty} F_{i}(A)}$. Thus, $A \subset \overline{\bigcup_{i=1}^{\infty} F_{i}(A)}$. The proof is complete.

From this result and Theorem 2.2 one deduces that the support of any invariant measure for $(\mathcal{F}, \rho)$ contains the set $\bar{P}$, the closure of the set of fixed points of finite compositions of members of $\mathcal{F}$. In the following theorem we prove that indeed the support of $\mu$ is equal to $\bar{P}$. 
Theorem 3.6.

Let $(X, d)$ be a complete metric space. Let $\mathcal{F}=\left\{F_{i}: i \in \mathbb{N}\right\}$ be a countable family of contraction mappings on $X$ and let $\rho=\left\{\rho_{i}: i \in \mathbb{N}\right\}$ be a probability sequence, i.e. $0<\rho_{i}<1$ and $\sum_{i=1}^{\infty} \rho_{i}=1$. If $\mu$ is an invariant measure for (F, $\rho$ ), then supp $\mu=\bar{P}$, where $P$ the set of fixed points of finite compositions of members of $\mathcal{F}$.

Proof. From Theorems 3.5 and 2.2 we have that $\bar{P} \subset \operatorname{supp} \mu$.

In order to prove the other inclusion, let us consider $x \notin \bar{P}$. We will prove that $x \notin \operatorname{supp} \mu$, by showing that there exists a neighbourhood of $x$ of zero $\mu$-measure. Since $x \notin \bar{P}$, let $\varepsilon=d(x, \bar{P}) / 2>0$. Let

$$
G=B(x, \varepsilon)=\{y \in X: d(x, y)<\varepsilon\} .
$$

We will prove that $\mu(G)=0$. Define, as before, the set $\bar{P}_{\varepsilon}$ as $\bar{P}_{\varepsilon}=\{y \in X: d(y, \bar{P})<\varepsilon\}$. Notice that $G \cap \bar{P}_{\varepsilon}=\emptyset$.

Now, let $i \in \mathbb{N}$ be arbitrary but fixed throughout the proof. Since $F_{i}$ is a contraction map whose fixed point is $x_{i}$ and whose contraction factor is $r_{i},\left\{F_{i}^{k}(x)\right\}_{k \in \mathbb{N}}$ converges to $x_{i}$ and $\left\{r_{i}^{k}\right\}_{k \in \mathbb{N}}$ converges to 0 . Then there exists $k=k(i)$ such that $r_{i}^{k}<1 / 2$ and $d\left(F_{i}^{k}(x), x_{i}\right)<\varepsilon / 2$. Further, again by the contractivity of $F_{i}$, we have that for any $j \in \mathbb{N}$, $F_{i}^{j}(G) \subset B\left(F_{i}^{j}(x), r_{i}^{j} \varepsilon\right)$, in particular $F_{i}^{k}(G) \subset B\left(F_{i}^{k}(x), r_{i}^{k} \varepsilon\right)$. Moreover, if $z \in B\left(F_{i}^{k}(x), r_{i}^{k} \varepsilon\right)$ then

$$
d(z, \bar{P}) \leq d\left(z, x_{i}\right) \leq d\left(z, F_{i}^{k}(x)\right)+d\left(F_{i}^{k}(x), x_{i}\right)<r_{i}^{k} \varepsilon+\frac{\varepsilon}{2}<\varepsilon .
$$

Therefore $F_{i}^{k}(G) \subset \bar{P}_{\varepsilon}$. Hence, $G \subset\left[\left(F_{i}^{k}\right)^{-1}\left(\bar{P}_{\varepsilon}\right) \backslash \bar{P}_{\varepsilon}\right]$.

To finish our claim, it will be enough to prove that $\mu\left(\left(F_{i}^{k}\right)^{-1}\left(\bar{P}_{\varepsilon}\right)\right)=\mu\left(\bar{P}_{\varepsilon}\right)$. Since $\bar{P}$ is an invariant set, we have $F_{n}\left(\bar{P}_{\varepsilon}\right) \subset \bar{P}_{\varepsilon}$ for all $n \in \mathbb{N}$. Consequently, for every $\left(i_{1} \ldots i_{k}\right) \in \mathbb{N}^{k}, F_{i_{1} \ldots i_{k}}\left(\bar{P}_{\varepsilon}\right) \subset \bar{P}_{\varepsilon}$ and therefore

$$
\mu\left(\bar{P}_{\varepsilon}\right) \leq \mu\left(F_{i_{1} \ldots i_{k}}^{-1}\left(\bar{P}_{\varepsilon}\right)\right) \quad \text { for all } \quad\left(i_{1} \ldots i_{k}\right) \in \mathbb{N}^{k} .
$$

Note that if for some $\left(i_{1} \ldots i_{k}\right) \in \mathbb{N}^{k}$ we had a strict inequality in the last equation, by the invariance of $\mu$ we would have that

$$
\mu\left(\bar{P}_{\varepsilon}\right)=\sum_{i_{1} \ldots i_{k}} \rho_{i_{1}} \ldots \rho_{i_{k}} \mu\left(F_{i_{1} \ldots i_{k}}^{-1}\left(\bar{P}_{\varepsilon}\right)\right) \geqslant \mu\left(\bar{P}_{\varepsilon}\right) .
$$

Therefore from equation (1) we must have $\mu\left(\bar{P}_{\varepsilon}\right)=\mu\left(F_{i_{1} \ldots i_{k}}^{-1}\left(\bar{P}_{\varepsilon}\right)\right)$ for all choices $\left(i_{1} \ldots i_{k}\right) \in \mathbb{N}^{k}$. In particular, by taking $i_{1}=\ldots=i_{k}=i$, we obtain $\mu\left(\bar{P}_{\varepsilon}\right)=\mu\left(\left(F_{i}^{k}\right)^{-1}\left(\bar{P}_{\varepsilon}\right)\right)$, and the proof is complete.

As noted before, the existence of the invariant measure depends only on the relatively weak condition $\sum_{i} \rho_{i} d\left(x_{1}, x_{i}\right)<\infty$, independently of the value of $r$. (For example if the set of fixed points of $F_{i}$ is bounded, the condition is already satisfied and guaranties the existence and uniqueness of an invariant measure.)

Our result shows, that in contrast to the case of invariant sets which may not be unique, if an invariant measure having bounded support exists, it is unique. Indeed, if $\mu$ is an invariant measure whose support is bounded, from our result it follows that the set of fixed points of members of $\mathcal{F}$ is bounded, which implies that $\sum_{i} \rho_{i} d\left(x_{1}, x_{i}\right)<\infty$ and, consequently we obtain the uniqueness of the invariant measure.

\section{Acknowledgements}

The authors acknowledge support from the following grants: PICT 2011-0436 (ANPCyT), PIP 01070 and 2008-398 (CONICET), UBACYT 20020100100502 (UBA) and PROICO 3-0412 (UNSL). 


\section{References}

[1] Bandt C., Self-similar sets. I. Topological Markov chains and mixed self-similar sets, Math. Nachr., 1989, 142, 107-123

[2] Barnsley M.F., Demko S., Iterated function systems and the global construction of fractals, Proc. Roy. Soc. London Ser. A, 1985, 399(1817), 243-275

[3] Falconer K.J., The Geometry of Fractal Sets, Cambridge Tracts in Math., 85, Cambridge University Press, Cambridge, 1986

[4] Falconer K., Fractal Geometry, John Wiley \& Sons, Chichester, 1990

[5] Hille M.R., Remarks on limit sets of infinite iterated function systems, Monatsh. Math., 2012, 168(2), 215-237

[6] Hutchinson J.E., Fractals and self-similarity, Indiana Univ. Math. J., 1981, 30(5), 713-747

[7] Kravchenko A.S., Completeness of the space of separable measures in the Kantorovich-Rubinshtein metric, Siberian Math. J., 2006, 47(1), 68-76

[8] Mattila P., Geometry of Sets and Measures in Euclidean Spaces, Cambridge Stud. Adv. Math., 44, Cambridge University Press, Cambridge, 1995

[9] Mauldin R.D., Infinite iterated function systems: theory and applications, In: Fractal Geometry and Stochastics, Progr. Probab., 37, Birkhäuser, Basel, 1995, 91-110

[10] Mauldin R.D., Urbański M., Dimensions and measures in infinite iterated function systems, Proc. London Math. Soc., 1996, 73(1), 105-154

[11] Mauldin R.D., Williams S.C., Random recursive constructions: asymptotic geometric and topological properties, Trans. Amer. Math. Soc., 1986, 295(1), 325-346

[12] Mihail A., Miculescu R., The shift space for an infinite iterated function system, Math. Rep. (Bucur.), 2009, 11(61)(1), 21-32

[13] Secelean N.A., The existence of the attractor of countable iterated function systems, Mediterr. J. Math., 2012, 9(1), $61-79$ 